\section{Mycobacterium avium duodenal infection mimicking Whipple's disease in a patient with AIDS}
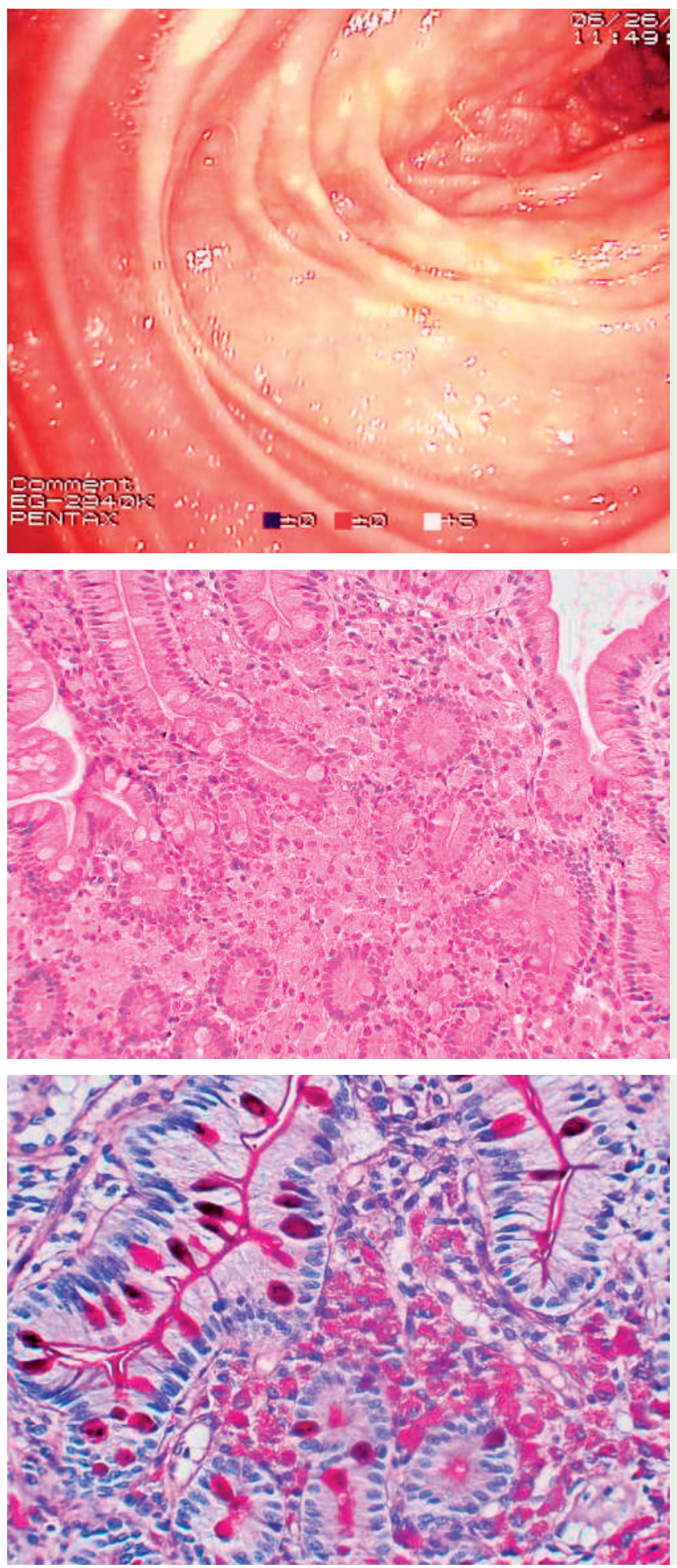

Fig. 1 Upper digestive tract endoscopy revealed a whitish nodular pattern in the second part of the duodenum.

Fig. 2 Histological view of duodenal biopsy tissue (hemato-eosin-safran stain, original magnification $\times 40$ ).

Fig. 3 Histological view of duodenal biopsy tissue after staining with periodic acidSchiff stain, original magnification $\times 400$ ).
A 45-year-old man was admitted to the intensive care unit for the management of septic shock secondary to infection with Salmonella enteritidis. The patient was also found to have Pneumocystis jiroveci and Mycobacterium avium pneumonia. Further work-up revealed him to be HIV-positive, with a CD4 count of 125/ $\mathrm{mm}^{3}$. The patient was started on a treatment regimen of cefotaxim, trimethoprim/sulfamethoxazole, prednisolone, clarithromycin, and ethambutol. He developed severe malnutrition (body mass index $13.2 \mathrm{~kg} / \mathrm{m}^{2}$, serum albumin $14 \mathrm{~g} / \mathrm{L}$ ) and was referred to our endoscopy center for placement of a percutaneous endoscopic gastrostomy.

Esophagogastroduodenoscopy revealed a whitish nodular pattern in the second part of the duodenum ( Fig. 1) and biopsies were obtained from this region. Pathological examination of these nodules showed extensive infiltration of the lamina propria with foamy histiocytes ( $\bullet$ Fig. 2); tests were positive for periodic acid-Schiff reagent ( Fig.3) and acid-fast staining (with diffuse bacillary inclusions, as shown in $\bullet$ Fig. 4) and negative for cytomegalovirus immunohistochemistry. Duodenal biopsy cultures were positive for M. avium and a diagnosis of M. avium gastrointestinal infection in a patient with recently diagnosed advanced AIDS was made. Despite intensive care and specific treatment with clarithromycin and ethambutol, the patient died of acute respiratory distress syndrome.

M. avium is the most common mycobacterium implicated in infections of the gastrointestinal tract in AIDS. It usually causes fever, diarrhea, and weight loss. Possible duodenal endoscopic findings are: normal mucosa, erythema, whitish exudate, and nodules mimicking Whipple's disease, this last appearance being the most distinctive feature [1-4]. Notably, this Whipple-like appearance is not only an endoscopic feature but is also seen on microscopic examination on standard staining (but not on acid-fast staining). A similar endoscopic pattern has also been described in a patient with gastrointestinal Mycobacterium genavense infection complicating advanced AIDS [5].

Endoscopy_UCTN_Code_CCL_1AB_2AZ_3AC 


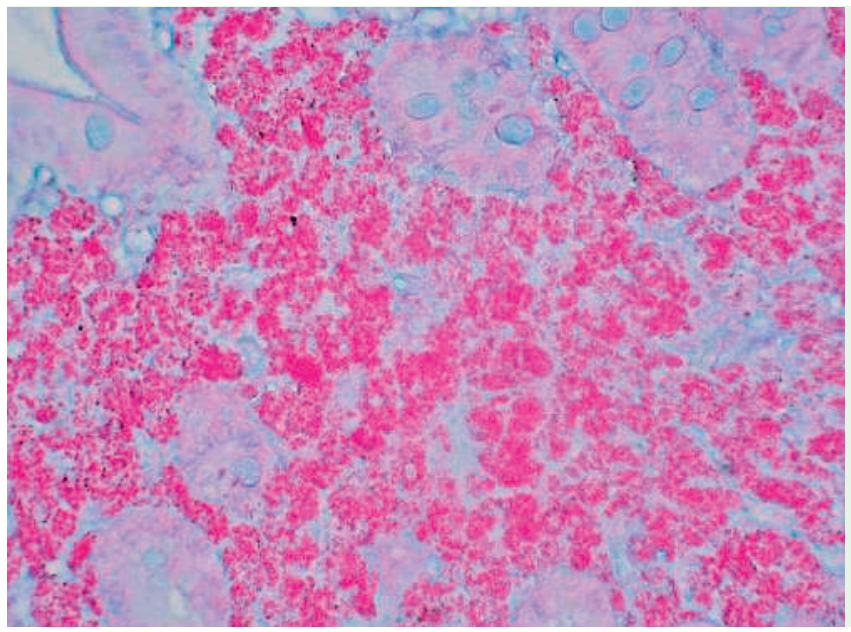

Fig. 4 Histological view of duodenal biopsy tissue after staining with acid-fast stain (original magnification $\times 200$ ).

\section{Dray ${ }^{1}$, K. Vahedi ${ }^{1}$, V. Delcey ${ }^{2}$, \\ A. Lavergne-Slove ${ }^{3}$, L. Raskine ${ }^{4}$, J.-F. Bergmann ${ }^{2}$, P. Marteau ${ }^{1}$}

1 Département de Pathologie Digestive, APHP, Hôpital Lariboisière, Université Paris 7, Paris, France

2 Service de Médecine, APHP, Hôpital Lariboisière, Université Paris 7, Paris, France

3 Service Central de Cytologie et Anatomie Pathologiques, APHP, Hôpital Lariboisière, Université Paris 7, Paris, France

4 Service de Bactériologie-Virologie, APHP, Hôpital Lariboisière, Université Paris 7, Paris, France
Bibliography

DOI $10.1055 / \mathrm{s}-2007-966483$

Endoscopy 2007; 39: 296-297

(c) Georg Thieme Verlag KG Stuttgart · New York . ISSN 0013-726X

\section{Corresponding author \\ X. Dray, MD}

Département de Pathologie Digestive

Hôpital Lariboisière

2 Rue Ambroise Paré

75475 Paris

France

Fax: +331-49-95-25-77

xavier.dray@Irb.aphp.fr 